\title{
You do not find your own face faster; you just look at it longer
}

\author{
Christel Devue ${ }^{1,2}$, Stefan Van der Stigchel ${ }^{2}$, Serge Brédart ${ }^{1}$, and Jan Theeuwes ${ }^{2}$ \\ ${ }^{1}$ Cognitive Science Department, Université de Liège, Belgium \\ ${ }^{2}$ Cognitive Psychology, Vrije Universiteit Amsterdam, the Netherlands
}

\begin{abstract}
Previous studies investigating the ability of high priority stimuli to grab attention reached contradictory outcomes. The present study used eye tracking to examine the effect of the presence of the self-face among other faces in a visual search task in which the face identity was task-irrelevant. We assessed whether the self-face (1) received prioritized selection (2) caused a difficulty to disengage attention, and (3) whether its status as target or distractor had a differential effect. We included another highly familiar face to control whether possible effects were self-face specific or could be explained by high familiarity. We found that the self-face interfered with the search task. This was not due to a prioritized processing but rather to a difficulty to disengage attention. Crucially, this effect seemed due to the self-face's familiarity, as similar results were obtained with the other familiar face, and was modulated by the status of the face since it was stronger for targets than for distractors.
\end{abstract}

\section{Keywords}

Allocation of attention, eye movement, familiar faces, self-face, visual search.

Correspondence to:

Christel Devue

Department of Cognitive Science

Université de Liège

Bd du Rectorat, 5 (Bât. B32)

B-4000 Liège

Belgium

Email: cdevue@ulg.ac.be

Tel.: +32[0]4 3662021

Fax : +32[0]4 3662859 


\section{Introduction}

When we open our eyes, we receive a large amount of visual information. Because our visual system has a limited capacity, selection must occur to prioritize important stimuli while ignoring less important ones. For almost fifty years, debates have opposed partisans of an early selection of attention (Broadbent, 1958), to whom this attentional selection concerns the gross stimulus features at an early stage of processing, to partisans of a late selection of attention (Deutsch \& Deutsch, 1963), to whom the attentional selection takes place after semantic processing of all the incoming information. To resolve these debates, highly important stimuli (e.g., one's own name) have been used, trying to determine whether they can be processed in conditions where they are supposedly unattended. These studies led to contradictory outcomes.

Since the publication of the seminal study by Moray (1959), the interest in investigating selfreferential material grew over the last few decades. Using a shadowing procedure during a dichotic listening task, Moray showed that participants were better in remembering that they heard their own name presented to one ear relative to other words while repeating aloud (.i.e. shadowing) a message presented to the other ear. However, these results were later criticized because there was no way to exclude the possibility that participants shifted their attention from time to time to the to-be-ignored ear, hence attending to the message displayed to that ear and therefore perceiving it (see Lachter, Forster, \& Ruthruff, 2004, that confirmed this criticism using a priming paradigm). Nonetheless, Wood and Cowan (1995) later replicated Moray's results with more careful control of temporal lapses of attention (i.e. they used the accuracy and speed of shadowing as online measures of attentional shifts). They found that participants who recalled their own name showed a disruption in shadowing shortly after the presentation of their own name but, importantly, not before or while the own name was presented. In a visual analog of Moray's paradigm in which participants were instructed to make a parity judgment on two digits flanking a to-be-ignored word (that was their own name on some trials), $80 \%$ of participants reported that they had seen their own name whereas they only recognized $68 \%$ of words presented in similar conditions (Wolford \& Morrison, 1980). In addition, the presence of the participant's own name affected response times relative to neutral words. According to the authors, their results argue in favour of a robust name effect (but see e.g. Bundesen, Kyllingsbaek, Houmann, \& Jensen, 1997). However, given the fact that the to-be-ignored stimuli were presented at fixation, they were located within the focus of attention and were presumably attended, preventing any strong conclusion in favour of a late selection theory of attention. Subsequently, other studies demonstrated that one's own name is particularly resistant to the attentional blink (Shapiro, Caldwell, \& Sorensen, 1997) and to repetition blindness (Arnell, Shapiro, \& Sorensen, 1999) during rapid serial visual presentations when compared to other names or nouns. In the inattentional blindness paradigm, the own name is also less subject to blindness than other names or frequent words (Mack \& Rock, 1998). Additionally, Mack and Rock (1998) showed that the own name pops out of a display of up to 12 items in a visual search task (but see Harris, Pashler, \& Coburn, 2004 who obtained search slopes that were not flat enough to claim that the own name pops out, although it was detected more rapidly than other names).

However, Bundesen et al. (1997) showed that one's own name does not automatically attract attention. In their experiment two white-coloured (to-be-ignored distractors) and two redcoloured (to-be-reported targets) names were presented. The own name was presented on some trials as target or as distractor. The own name presented as distractor did not cause more interference than other names but participants were more accurate in reporting their own name 
presented as targets than in reporting targets from display only having other names. However, a control experiment showed that this advantage for the own name presented as target was not attentional, but rather reflected a better identification of the participant's own name compared with other names. Other studies later demonstrated that one's own name summons attention when participants are set to identify target names but not when participants are set to find the colour of a target (Kawahara \& Yamada, 2004). Similarly, in a Stroop-like task, the own name attracted attention if presented centrally within the participant's attentional focus but when presented peripherally, it attracted attention only when it was task-relevant but not when it was task-irrelevant (Gronau, Cohen, \& Ben-Shakhar, 2003).

More recent studies have examined the attentional properties of another self-referential stimulus, the self-face, and also found contrasting results. For instance, Laarni et al. (2000) found that only $18 \%$ of participants reported the presence of their own face in the background while they were performing a matching task on two faces presented at foreground. Similar results were obtained with a famous face (the Finnish President). Conversely, Brédart, Delchambre and Laureys (2006) found that the self-face flanking a classmate's name in a face-name interference paradigm produced a stronger interference on the processing of this name than in the reverse situation (i.e., when a classmate's face flanked the participant's own name). This suggests that the self-face also has some attention-grabbing capacity. Similarly, Tong and Nakayama (1999) showed that the self-face was more quickly detected among distractors than strangers' faces, even when presented in atypical orientations and after hundreds of trials.

In sum, existing data do not unanimously support the late selection view (Deutsch \& Deutsch, 1963) that suggests that stimuli of particular importance with particular meaning (such as selfreferential material) would attract attention. Nonetheless, a careful examination of the different paradigms and conditions used in previous studies might help resolving some of the discrepancies between the studies. It seems that "self-effects" were mostly found when selfreferential stimuli were presented centrally, within the participant's focus of attention (see e.g., Arnell et al., 1999; Gronau et al., 2003-Experiment 1; Shapiro et al., 1997; Wolford \& Morrison, 1980). In that case however, it is difficult to conclude that self-referential stimuli capture attention since they were already within the focus of attention. In such a situation, their processing was presumably inevitable when attending to the target items because they were also located centrally. By contrast, when self-referential stimuli were presented outside the focus of attention, they produced specific effects when their processing was somehow related with the task at hand (see e.g., Brédart et al., 2006; Gronau et al., 2003-Experiment 3) but they generally failed to elicit specific performance in conditions that rendered their processing completely irrelevant (see e.g. Bundesen et al., 1997; Gronau et al., 2003Experiment 2; Laarni et al., 2000).

Overall, this might suggest that, in particular conditions, rather than capturing attention selfreferential stimuli seems to hold attention. In addition, the extent to which the distractor does or does not share some properties (e.g., in terms of spatial location, of semantic relationship, etc.) with the target seems important. Therefore, on the one hand, the lack of coherence in previous studies could be partly due to a lack of differentiation between the capture and retention components of attention and, on the other hand, to the properties of the selfreferential stimuli in the context of a given experiment.

As a consequence, in the present study, we used the eye tracking technique to investigate more precisely the way in which attention is allocated when the self-face is presented among unfamiliar faces during a visual search task. Typically, in a visual search task participants are 
required to divide attention over multiple elements in the display ensuring that attention cannot be focused on one element. Coupled with eye movements recording, it allows an exhaustive examination of both early (e.g. attentional capture reflected by a direct saccade towards the stimulus) as well as later (e.g. preferential allocation or shifts of attention reflected by following saccades and fixations) stages of attentional allocation (see e.g. Weierich, Treat, \& Hollingworth, 2008). We put three main questions: (1) Do our eyes go faster to the self-face than another face? In other words, is the self face prioritized in visual search? ; (2) Once attending a face, do our eyes stay longer on the self-face than on another face? In other words, does the self-face hold attention? ; (3) Are these potential effects dependent on the status of the self-face as target or distractor? In order to disentangle "selfeffects" from mere familiarity effects, we included another highly familiar face (a friend's face) in the experiment. We designed a visual search task in which participants searched an array of familiar and unfamiliar faces looking for a face with a particular configuration resulting from the pronunciation of a specific sound. The task implied processing facial features but without the need to process the facial identity. Therefore, the familiarity and identity of the faces were completely task-irrelevant but their processing remained possible in the conditions of the task. Moreover, the task-relevant features were neutral with respect to emotional content in order to prevent any effect of other confounding emotional variables.

\section{Method}

Participants. Twenty-two participants (four males) were recruited by gender-matched pairs so that each of them served as the friend for the other participant. They had to know each other for at least 6 months (mean relationship duration $=20$ months), were engaged in a positive relationship and saw each other on a regular basis (i.e., they were classmate or friends).

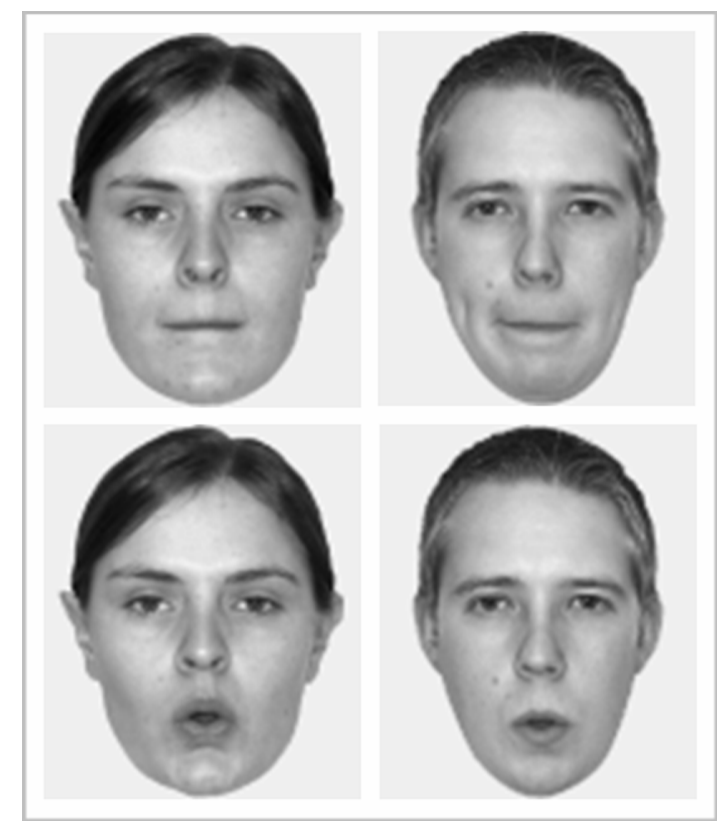

Figure 1. Sample face stimuli showing a [m] sound (top) and an [o] sound (bottom).

Stimuli. Twenty-two individuals (11 females and 11 males) had been photographed to be used as unfamiliar faces, in a frontal position while pronouncing a [m] and an [o] (i.e., two easily producible and differentiated phonemes, see Figure 1). Each participant in the experiment was also photographed in similar conditions, the two pictures being used as "self-face" for him/herself and as "friend's face" for his/her friend. All pictures were converted in greyscales. 
Hair below the ear lobes and neck were removed so that all faces had an overall oval shape. Faces were placed in a uniformed light grey background and resized to subtend 2.9 degrees in height and around 2.1 degrees in width. The self-face was presented in a mirror orientation.

Procedure. Participants were individually tested in a dim-lighted room. They were maintained at a $75 \mathrm{~cm}$ distance from the computer screen by means of a chinrest. Eye movements were measured with an Eye Link II eye tracking system with $500 \mathrm{~Hz}$ sampling rate. Participants were instructed to judge whether a target face was present or absent in a 6-faces display (see Figure 2) by means of two response keys. For half of the participants the target face displayed a [m] and remaining distractors displayed an [o]. The reversed situation was presented to the remaining half of participants. Participants received no instruction about the presence of familiar faces. Each trial began with a fixation cross that participants were instructed to stare at until the presentation of the faces. After $500 \mathrm{~ms}, 6$ faces positioned on a virtual circle at 8.3 degrees around fixation appeared until a response was made (up to 3 seconds), followed by a blank of $1000 \mathrm{~ms}$. If participants moved their eyes away from fixation (1.3 degrees) before the pictures onset, they heard a "beep" sound. They received a visual feedback in case of incorrect or too slow responses.

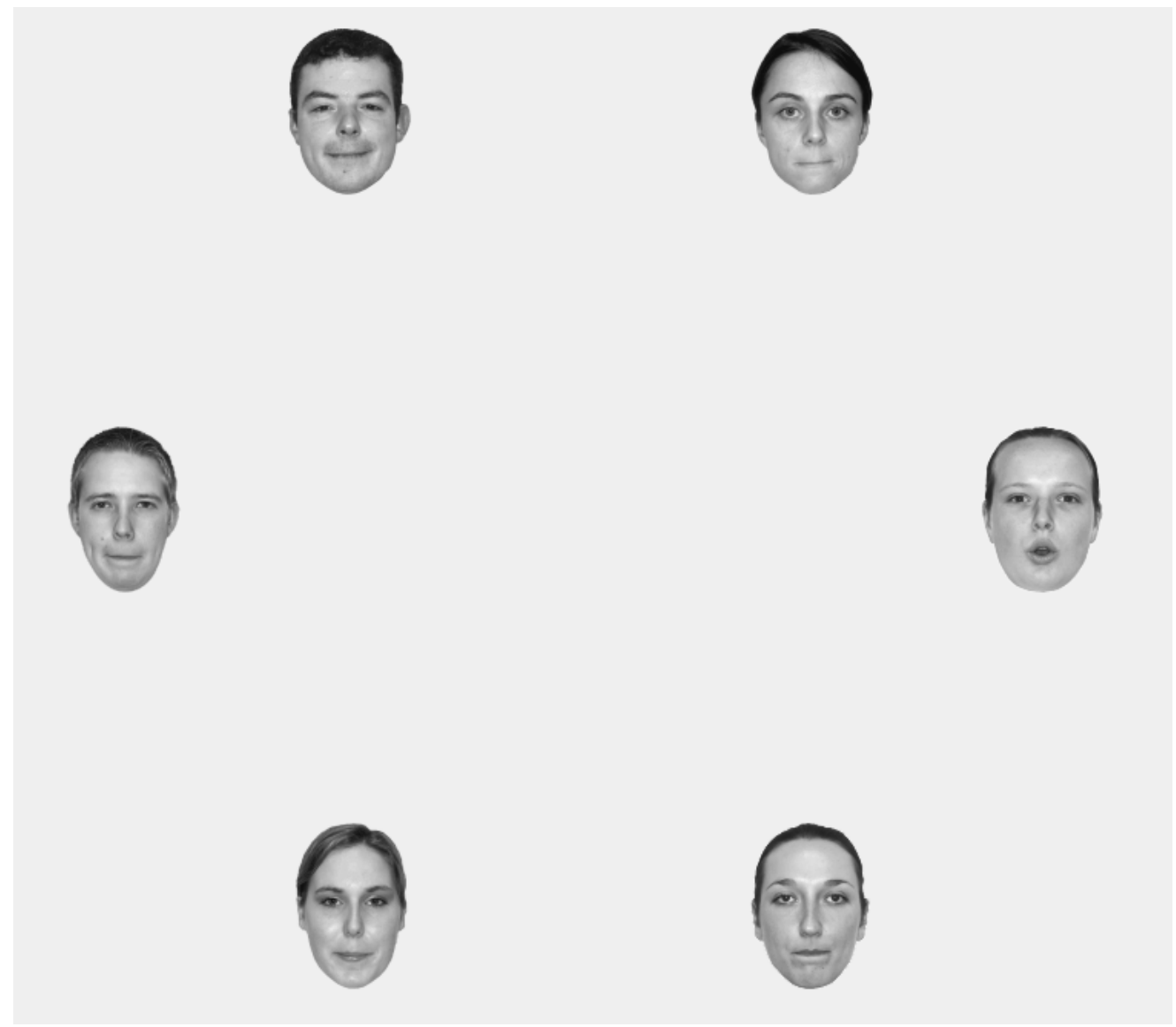

Figure 2. Illustration of a 6-faces display in which a target is present ([o] target among [m] distractors).

The test included 288 trials presented in a random order. A target was present in $50 \%$ of these trials. Each familiar face was presented a total of 72 times (the self-face on $25 \%$ of trials and the friend's face on $25 \%$ of trials) distributed as follows: half the time in the absent target condition (36 times) and every other time in the present target condition (36 times). In that latter case, each familiar face was the target once out of 6 (6 times) and it was a distractor the 
5 remaining times out of 6 (30 times). Each familiar face was always presented along with 5 unfamiliar faces. Hence, the processing of the familiar faces was completely irrelevant for the task and the presence of one of these faces was totally unpredictable of the correct response.

Displays were only composed of unfamiliar faces in the remaining $50 \%$ of trials that were distributed as follows: 72 trials with a target present and 72 trials with no target. The unfamiliar faces were picked up randomly among the set of 22 unfamiliar faces with the constraint that two identical faces could not appear within the same trial. Each individual unfamiliar face had thus the same probability to be presented as each familiar face (in $25 \%$ of trials) and the same probability to be presented as a target $(2.1 \%)$ or as a distractor $(22.9 \%)^{1}$.

Before the test, participants performed a 48-trials training session composed of 6 additional unfamiliar faces that were not presented during the test.

Design and data analysis. We first examined the effect of the Condition (target present vs. target absent) and of the inclusion of a familiar face within the display (Display type: selfface, friend's face, all unfamiliar faces) on mean reaction times (RTs) and on mean number of saccades. The mean number of saccades was the number of eye movements necessary to judge correctly whether the target was present or absent (from the display onset until a correct response was given). These analyses determined whether the presence of a familiar face within the display interfered with the ongoing task by comparison with a condition in which only unfamiliar faces were presented.

In addition we assessed, for target faces, the effect of the Face's identity (self-face, friend's face, unfamiliar face) on the first time to arrive at this very face. The first time to arrive at the target corresponded to the delay between the display onset and the time point where the eyes landed for the first time on the relevant face. Note that, here, the "unfamiliar face" identity represents "pure" unfamiliar trials in which no familiar face was present within the display. This analysis assessed the existence of a prioritization of processing for the self-face. Finally, we examined the effect of the Face's identity and of its Status (target vs. distractor) on total glance duration; a measure defined as the total time spent fixating the face. The unfamiliar distractor condition was computed by choosing one unfamiliar face at random among the possible unfamiliar distractor faces. This analysis tested whether the self-face holds attention more than another face. In order to properly compare targets and distractors, only target present trials were taken into account in this analysis. Moreover, only trials in which a correct response was given and in which the relevant face was fixated were included in these two analyses.

Trials with anticipatory eye movements (latencies shorter than $80 \mathrm{~ms}$ ) were excluded from analyses.

\section{Results}

We discarded data of two participants because those participants only had $20 \%$ and $23 \%$ of analysable data (i.e., trials without too early saccades or errors) and we did not include data of one participant whose response time and accuracy deviated more than $2.5 S D$ from the mean of the sample.

RTs. A two-way repeated measures ANOVA with Condition (target present vs. absent) and Display type (i.e., the face included in the display: self-face, friend's face, all unfamiliar faces) as factors was conducted on RTs. Condition had a significant effect, $F(1,18)=386.34$; 
$p<0.001$, with faster responses when the target was present $(M=1373 \mathrm{~ms}, S D=254 \mathrm{~ms})$ than when it was absent $(M=1878 \mathrm{~ms}, S D=370 \mathrm{~ms})$. Display type also had a significant effect, $F(2,36)=3.63 ; p<0.05$. Planned comparisons indicated that RTs were slower when the self-face $(M=1642 \mathrm{~ms}, S D=276 \mathrm{~ms})$ and when the friend's face $(M=1630 \mathrm{~ms}, S D=$ $252 \mathrm{~ms})$ were presented than when only unfamiliar faces $(M=1604 \mathrm{~ms}, S D=226 \mathrm{~ms})$ were presented, respectively $p=0.018$ and $p=0.049$. By contrast, RTs were not significantly different when the self-face and when the friend's face were presented, $p=0.47$. There was no interaction between Condition and Display type, $F<1$ (see Figure 3A).

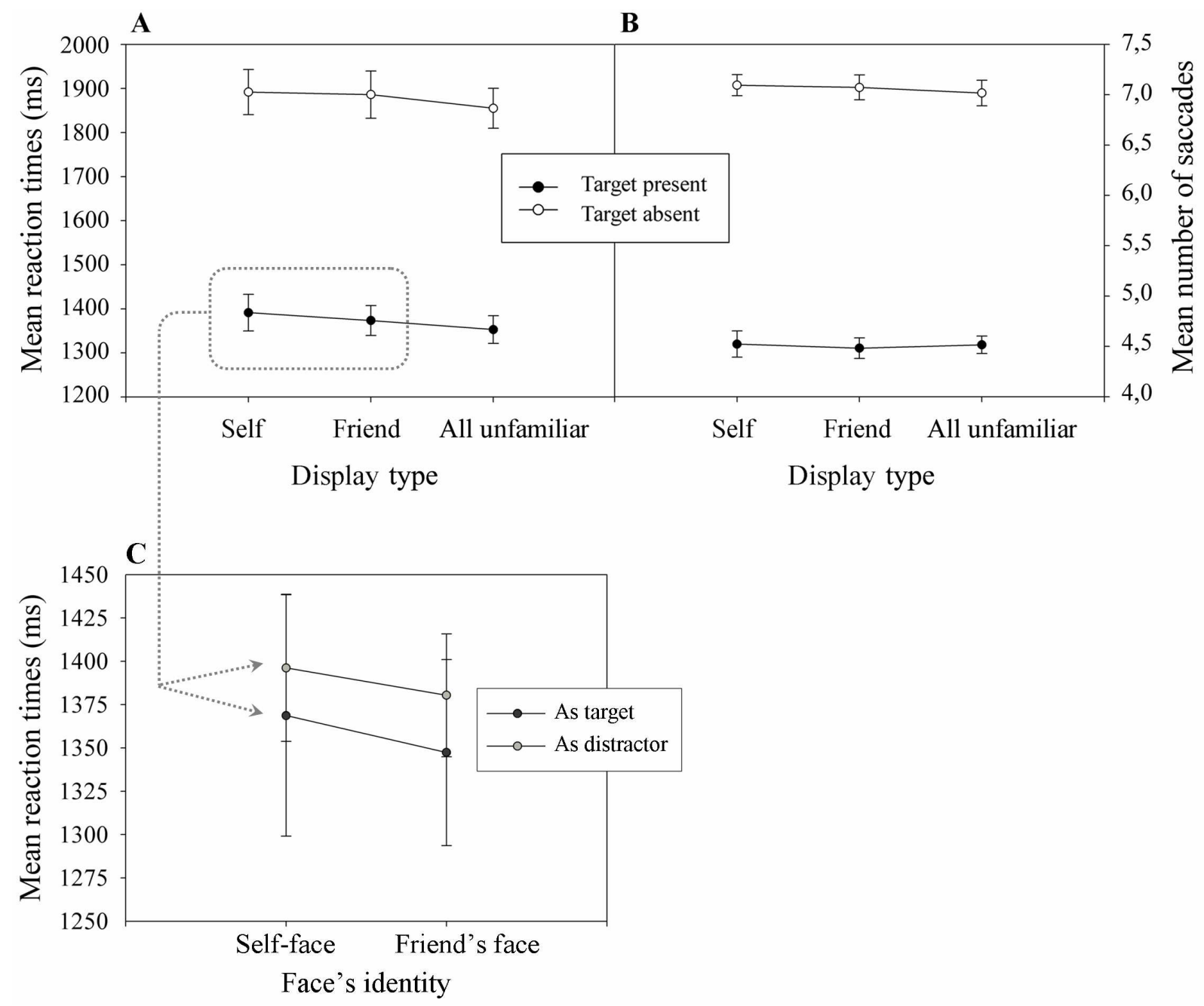

Figure 3. Mean reaction times (A) and mean number of saccades necessary to judge whether the target was present or absent $(B)$ as a function of the Condition and of the face contained in the display (Display type). Mean reaction times $(C)$ in the target present condition as a function of the Status (target vs. distractor) of the familiar faces present within the display.

Error bars represent SEM.

Moreover, to specify further the interference caused by the familiar faces as a function of their status in the target present condition, we conducted a two-way repeated measures ANOVA with Status (target vs. distractor) and Face's identity (self-face vs. friend's face) as factors. There was no significant effect and no interaction, all $F_{\mathrm{s}}<1$ (see Figure 3C).

Mean number of saccades. We also conducted a 2 (Condition) X 3 (Display type) ANOVA on the mean number of saccades. Condition had a significant effect, $F(1,18)=573.56 ; p<$ 
0.001, as fewer saccades were necessary to respond when the target was present $(M=4.51$, $S D=0.69)$ than when the target was absent $(M=7.06, S D=0.84)$. Display type had no significant effect, $F<1$, and there was no interaction, $F<1$ (see Figure 3B).

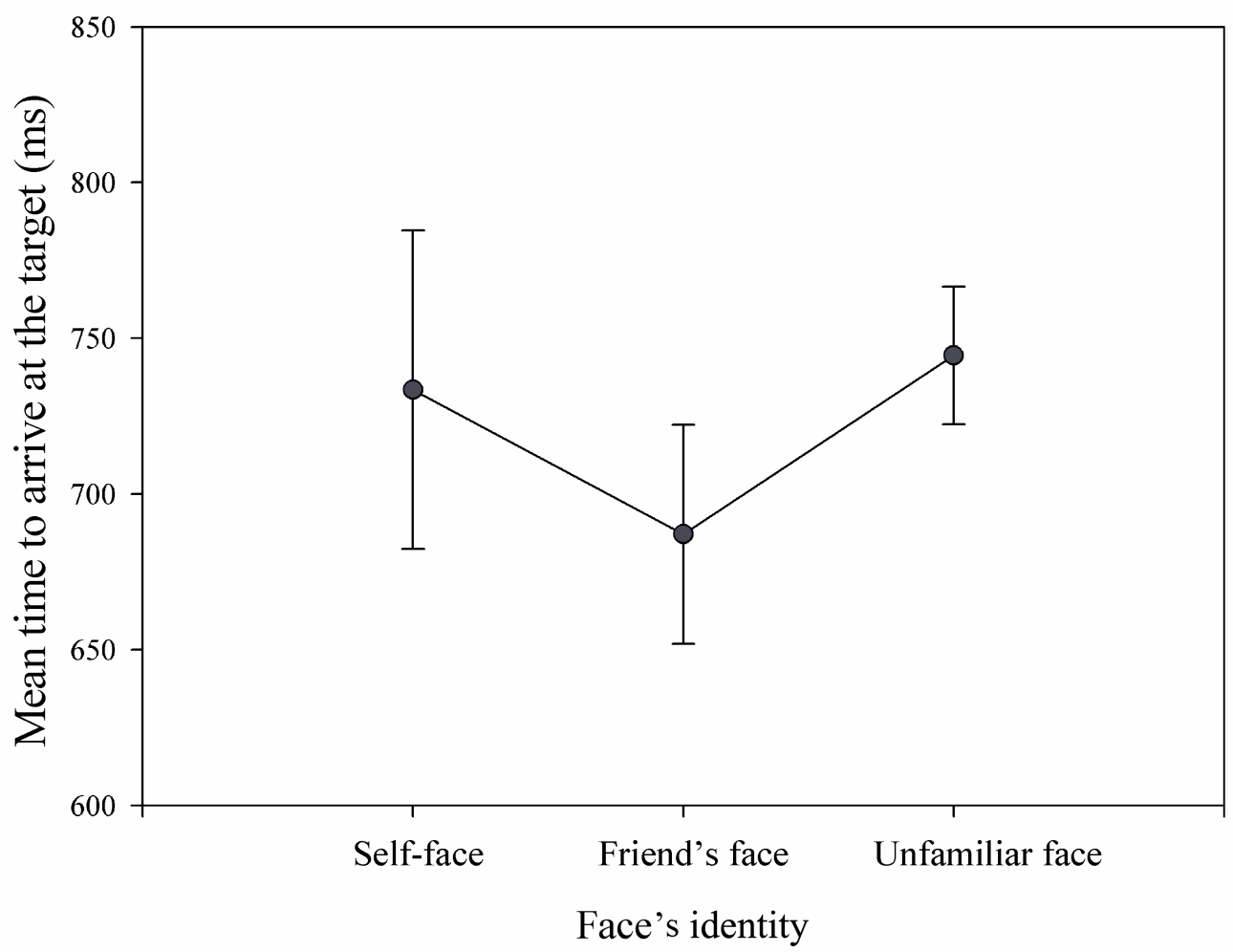

Figure 4. Mean time to arrive at a target face as a function of its Identity. Error bars represent SEM.

First time to arrive at the target. In this analysis we determined the first time participants arrived with their eyes at the self-face $(M=733 \mathrm{~ms}, S D=223 \mathrm{~ms})$, a friend's face $(M=687$ $\mathrm{ms}, S D=153 \mathrm{~ms})$ or an unfamiliar face $(M=744 \mathrm{~ms}, S D=96 \mathrm{~ms})$ as targets. A one-way repeated measures ANOVA showed no effect of Face's identity, $F<1$ (see Figure 4).

Total glance duration. A two-way repeated measures ANOVA was performed to determine whether there was a difference in glancing at the self-face, a friend's face and an unfamiliar face (Face's identity) depending on whether it was a target or a distractor (Status). Face's identity affected the total glance duration, $F(2,36)=7.74 ; p<0.002$. Planned comparisons indicated that the self-face $(M=380 \mathrm{~ms}, S D=121 \mathrm{~ms})$ and the friend's face $(M=358 \mathrm{~ms}$, $S D=148 \mathrm{~ms})$ were fixated longer than an unfamiliar face $(M=323 \mathrm{~ms}, S D=67 \mathrm{~ms}), p<$ 0.001 and $p<0.05$, respectively. The two familiar faces did not differ between each other, $p$ $=0.14$. Status of the face significantly influenced the total glance duration, $F(1,18)=146.46$; $p<0.001$, with target faces $(M=484 \mathrm{~ms}, S D=207 \mathrm{~ms})$ being fixated longer than distractor faces $(M=224 \mathrm{~ms}, S D=58 \mathrm{~ms})$.

The interaction between Face's identity and Status was significant, $F(2,36)=3.64 ; p<0.05$. Planned comparisons indicated that the self-face as target was fixated longer than unfamiliar target faces, $p<0.001$, and that the friend's face as target only tended to be fixated longer than unfamiliar target faces, $p=0.072$. The two familiar faces as targets were not differentially glanced, $p=0.17$. The self-face as distractor was also fixated longer than an unfamiliar distractor face, $p<0.02$. The other comparisons between identities did not reach 
significance, all $p \mathrm{~s}>0.10$. All faces were fixated longer when they were targets than when they were distractors, all $p$ s $<0.001$ (see Figure 5).

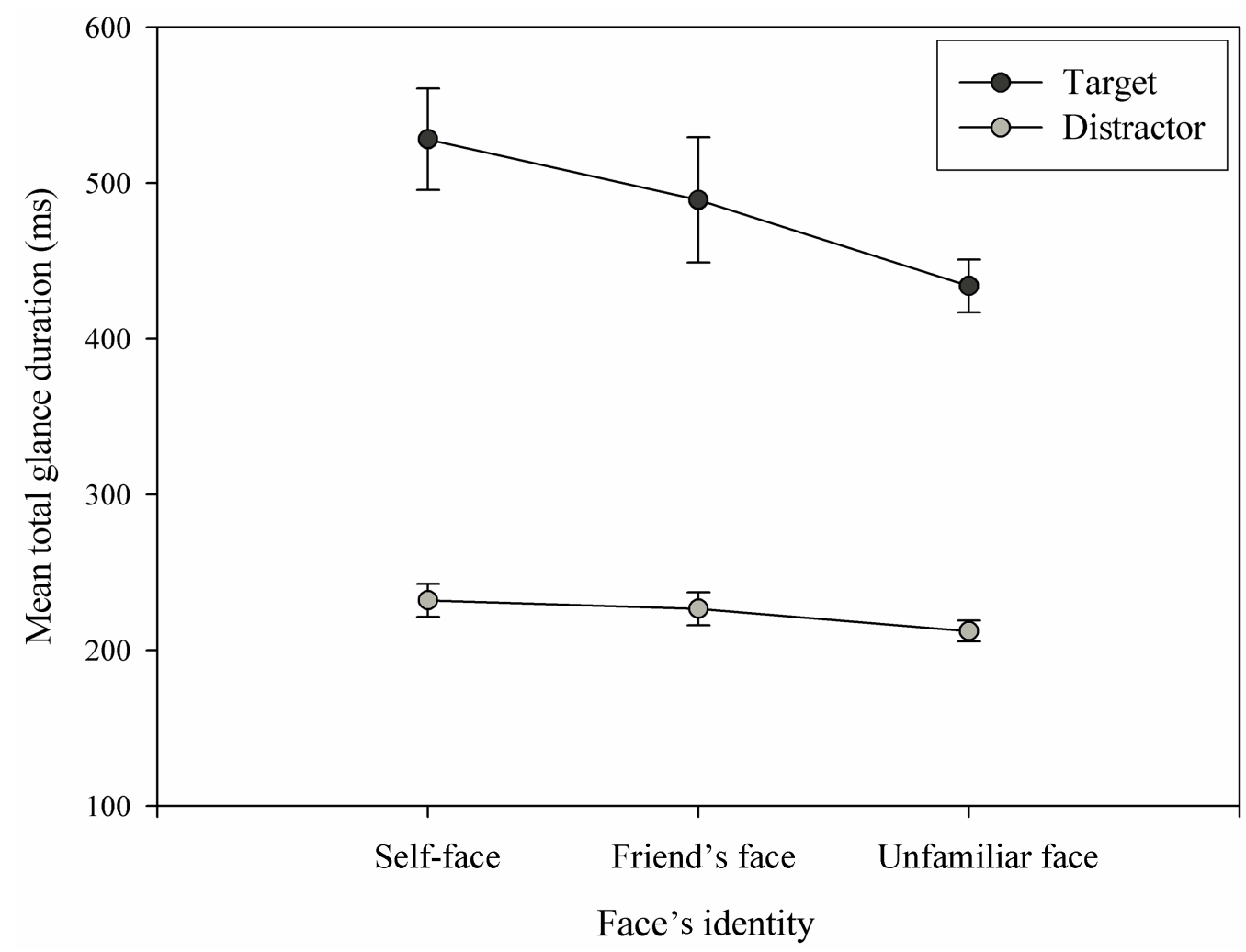

Figure 5. Mean total glance duration on a face as a function of its Status (target vs. distractor) and Identity. Error bars represent SEM.

\section{Discussion}

The first finding is that search in our task was performed in a slow and serial manner (see Figure $3 \mathrm{~A}$ and $\mathrm{B}$; a movie showing a sample of eye movements is also presented as supplementary material. (Note that for privacy reasons in this movie the eyes have been removed. However this was not the case in the experiment). As is clear from Figure 3, in target present trials it took about $1400 \mathrm{~ms}$ and 4.5 saccades to respond. In target absent trials RTs were about $1900 \mathrm{~ms}$ and the search took about 7 saccades. So in other words, there were on average 2.5 more saccades in target absent relative to target present trials and all 6 faces were serially inspected before a response could be generated in target absent trials. Importantly and in line with previous observations (Brédart et al., 2006), the presence of the self-face in the display interfered with the ongoing task as participants responded faster to displays in which only unfamiliar faces were presented compared to displays in which the self-face was present. This effect is even more striking that even if the processing of faces was necessary to the task, the face identity was completely task-irrelevant. Critically, here the presentation of another familiar face also caused a distraction arguing in favor of a familiarity effect rather than a "self effect".

Crucially, the current eye movement data allow us to directly determine the cause of this interference. Indeed, reaction times data alone could not disentangle whether this effect results from the processing of the self-face being prioritized (independently of its relevance for the task) or from the self-face holding attention only once it is located within the 
participant's focus of attention. So, we first examined whether the self-face automatically summons attention in comparison with an unfamiliar face and another highly familiar face. We assessed the delay between the onset of the display and the moment a saccade landed for the first time on a face. We found no effect of the face's identity as the time to arrive on a face was similar for all three types of faces. Note however that our control study clearly indicates that the faces were readily recognizable from the central fixation point. In other words, the absence of attentional prioritization cannot be due to insufficient perceptual acuity when fixating the middle. Inconsistent with previous findings regarding the own name (Mack \& Rock, 1998; Wolford \& Morrison, 1980) or the self-face (Brédart et al., 2006; Tong \& Nakayama, 1999), current results thus clearly indicate that the self-face does not benefit from attentional prioritization among other faces. Note that in the current study it is critical that we examined whether one's own face would be prioritized among other faces. Previous studies have already established that a face among non-face objects receive attentional priority (e.g., Ro, Russell, \& Lavie, 2001; Theeuwes \& Van der Stigchel, 2006). In the current study we asked the question whether the features that make up your own face would receive priority over faces which do not contain one's own facial features. Moreover, to establish whether such prioritization (if it would have occurred) would be truly bottom-up we ensured that task that observers had to perform had nothing to do with face identity as such.

Even though our findings showing no prioritization of the own face is inconsistent with some studies (Mack \& Rock, 1998; Wolford \& Morrison, 1980; Brédart et al., 2006; Tong \& Nakayama, 1999), other have reported similar findings showing that one's own name (Bundesen et al., 1997; Harris et al., 2004) or the self-face (Laarni et al., 2000) does not summon attention. Therefore, these discrepancies between previous findings as well as the present interference effect could be explained by a difficulty to disengage attention from selfreferential/highly familiar stimuli once they are attended, rather than by an attentional prioritization.

This is what we examined in a second analysis of eye movement data. Indeed, total glance duration data permit us to directly assess whether the self-face holds attention in comparison with an unfamiliar face or with another highly familiar face. We found that overall the selfface and the friend's face were fixated longer than unfamiliar faces. By contrast, the total glance durations on the self-face and on the friend's face were similar. Therefore, even if familiar faces were not prioritized in visual search (i.e. they were not found faster), they were fixated longer once they were located in the participant's focus of attention. In other words, it was more difficult to disengage attention from those familiar faces by comparison with less familiar faces. Hence, present data suggests that highly familiar stimuli could just benefit from a preferential allocation of attention instead of a bottom-up ability to capture attention.

We hypothesised that a lack of distinction between the capture and retention components of the deployment of attention could resolve apparent contradictions in previous studies. Our data thus seem to support this view and indicate that some "self-effects"2 found previously might also be due to a difficulty to disengage attention rather than to a prioritized processing of self-referential stimuli. Indeed, those effects have been found when self-referential stimuli that were irrelevant to the ongoing task were located within the focus of attention (Gronau et al., 2003; Wolford \& Morrison, 1980) but not when located outside the focus of attention (Bundesen et al., 1997; Gronau et al., 2003; Laarni et al., 2000), suggesting that they do not summon attention. In other words, discrepancies between previous studies could be explained in terms of attentional disengagement rather than in terms of the ability to attract attention automatically. To put it clearly, "self-effects" found in previous studies could be due to a retention of attention by important stimuli in cases where they happened to be within the 
participant's focus of attention (e.g. if their location coincided with the location of the target). The absence of specific effects of self-referential stimuli might actually have been observed in cases where they were located outside the focus of attention and never attended (e.g. for the presentation time was too short to allow attention shifting towards peripheral irrelevant items). Importantly, similar results in terms of attentional retention have been found with other types of important stimuli (i.e. threatening words, see Fox, Russo, Bowles, \& Dutton, 2001).

We also hypothesised that the extent to which task-irrelevant self-referential stimuli share some properties with the task-relevant features might also be important in determining specific attentional properties of these stimuli. Present data support this claim since the preferential allocation observed for familiar faces was modulated by the stimulus status as target or as distractor (as revealed by the Face's identity by Status interaction on total glance duration) even though face identity was completely task-irrelevant. The effect of identity on total glance duration was stronger when faces were targets than when they were distractors. More specifically, the self-face was fixated longer than unfamiliar faces both when it was a target and when it was a distractor. The results for the friend's face were slightly different from that of the self-face. Indeed, the friend's face tended to be fixated longer than unfamiliar faces when it was a target but did not differ from unfamiliar faces when it was a distractor. This indicates stronger effects for the self-face than for other highly familiar faces. Note however, that it would be too strong to argue in favour of a real "self-effect" because the friend's face overall behaved more like the self-face than like unfamiliar faces. Rather, this could be due to a familiarity effect because one may be more familiar with one's own face than with the face of a friend ${ }^{3}$.

In any case, this modulation of attentional retention by the status of the face is in line with findings of previous studies. For instance, it has been shown that self-referential stimuli elicited specific effects when their processing was somehow related with the task at hand (Brédart et al., 2006; Gronau et al., 2003; Kawahara \& Yamada, 2004; Shapiro et al., 1997) but not when their processing was totally irrelevant for the task and when they never shared relevant properties with the target items (e.g. Bundesen et al., 1997). Kawahara and Yamada (2004) introduced the notion of an "input filter" to account for such effects. According to them, the input filter prepares the observer to optimise his/her performance on a task and this will determine whether a specific feature of a stimulus will attract (or "retain", we could now add) attention or not. They posit that in Bundesen et al.'s (1997) study, which used masked presentations of 4-name displays (i.e. two red targets and two white distractors), the own name did not attract attention when presented as a distractor because participants tuned their input filters to pass the target red items. As a consequence, the white items (and therefore the own name as distractor) were not processed. In two experiments, Kawahara and Yamada (2004) used an adapted version of the attentional blink paradigm and showed consistent with their hypothesis that the own name only attracted attention when participants were set to identify target names whereas it did not when participants were set to find a target colour. Similarly, in the study by Laarni et al. (2000), the task-irrelevant presentation of the self-face did not interfere with the main task. In this study, the self-face appeared in the background with target items presented in the foreground (see also Gronau et al., 2003 for similar findings with one's own name). So in that case, the input filter probably included spatial location.

On the basis of the notion of an input filter, we can explain our results in more general terms. First, only task-relevant features (e.g., here the shape of the mouth, the red colour in Bundesen et al., 1997) would be selected and processed. At this stage of processing, when the task-relevant features do not match those defining the target (e.g. the shape of the mouth 
displaying an "M" whereas the target is an "O" mouth), other task-irrelevant features of the item (e.g., those determining facial identity in the current study or the meaning of the white words in Bundesen et al., 1997) will be processed to a lesser extent. This might explain the weaker effect of the familiar faces as distractor in the current study as well as the absence of any effect of distractors in other studies where distractors' features never matched the primary task (e.g., the white items in Bundesen et al., 1997). When the task-relevant features match those defining the target (e.g. when the shape of the mouth is an "O") then attention engages on this stimulus before the participant decides that she has found the target. This engagement of attention on target items is reflected by the current data by showing that targets were overall fixated about twice as long as distractors and might correspond to a checking process before the response is given. This longer processing of the target items would allow a deeper processing of this stimulus and as a consequence of its task-irrelevant features (e.g. here, the identity of the face). These task-irrelevant features could in turn have a distractive power triggering an even longer engagement of attention when they pertain to a highly familiar stimulus or a stimulus having a high adaptive importance (e.g. threatening stimuli).

It has to be mentioned that our results somehow contrast with those of another visual search study showing that one's own face is detected faster than other faces (Tong \& Nakayama, 1999; see also Mack \& Rock's, 1998, visual search study with one's own name, but see Harris et al., 2004). However, in the present study the target was defined on the basis of part-based information (i.e., the facial configuration resulting from the pronunciation of a given speechsound, so mostly the shape of the mouth). By contrast, in Tong and Nakayama (1999)'s study the participant's own face was the target participants had to search for and it was presented among strangers' faces. In other words, the search concerned facial identity. These opposite patterns of results (i.e. increased reaction times in one case and reduced in the other) could again be explained by the different attentional set of the participants. Since identity information is contained in the configural face information it remains possible that the selfface and the familiar face would received prioritized processing if search would concern all features of a face resulting in a configural attentional set (e.g., search for the face with features moved inwards towards the centre of the face $)^{4}$. Nonetheless, the aim of the present experiment was to assess the existence of a bottom-up prioritization of the self-face. So it was on purpose that the task set was not identity-related information. Indeed, to establish true bottom-up prioritization, what potentially could capture attention (in this case, the face identity) should be different from what people look for (in this case a facial configuration). For example, in Theeuwes, Hahn, Irwin and Kramer (1998) people searched for a red coloured disk while the eyes of the observers were captured by an abrupt onset which was completely irrelevant for the task at hand. Similarly, in the present experiment participants searched for a facial configuration (i.e., the shape of the mouth) while we examined whether identity information (i.e., their own face) would receive attentional priority. Our results clearly indicate that it did not. Note however that it is also unlikely that people do not process the whole face when they are looking for the configuration of the mouth. Previous research has demonstrated that once an object is attended it results in the mandatory processing of all attributes of that object (e.g., Kramer \& Jacobson, 1991).

Finally, in general, the present results suggest that attention and foveal inspection are necessary to discriminate the fine features defining a target when other complex visual stimuli are present (see also Lachter et al., 2004). Therefore, they support an early selection theory of attention (see Broadbent, 1958) rather than a later selection theory of attention (Deutsch \& Deutsch, 1963). Similar conclusions have been reached on the basis of other eye-movement studies using scenes (see e.g., Gareze \& Findlay, 2007; Underwood, Templeman, Lamming, $\&$ Foulsham, 2008). For instance, using a comparative search between two images, 
Underwood et al. (2008) showed that incongruent objects within the context of a scene were fixated faster than congruent objects, indicating that the former could be somehow detected before being fixated. Nonetheless, those objects received this first saccade only after the scene was presented for several seconds and a fixation to the object was necessary for this object to be identified.

In conclusion, we found that a unique and distinctive self-referential stimulus such as the selfface is not systematically prioritized in comparison to another highly familiar face or even by comparison with a less important unfamiliar face. Moreover, we demonstrated for the first time that once the self-face is fixated, it holds attention as it seems more difficult to disengage attention from it than from a less familiar face. Importantly however, this effect was dependent upon the status of the face as target and similar effects were observed with another highly familiar face.

\section{Footnote}

${ }^{1}$ To ensure that acuity from fixation was sufficient to recognize the faces at this eccentricity we conducted a control experiment with 8 other participants. The design was the same as in the main experiment except that one single face was presented at one of the 6 possible locations. The five other locations were occupied by grey ovals. The presentation time was reduced to $180 \mathrm{~ms}$ to prevent participants from making effective saccade and fixating the face. The faces were either the participant's face, one familiar face or 2 unfamiliar faces (displaying an " $\mathrm{M}$ " in a half of the trials and an "O" in the other half). Each person's face appeared in $25 \%$ of trials. Participants were to perform a 3-AFC (i.e. "me", "friend" or "unfamiliar face") identification task. The correct identification rates were $94 \%, 95 \%$ and $96 \%$, respectively, indicating that faces were readily recognizable at this eccentricity.

${ }^{2}$ For the sake of clarity we refer to the results of previous studies by using the term "selfeffects" because it was generally those effects that were under investigation. Note however that in some cases those results might be subtended by familiarity as in the present experiment. Indeed, in some previous studies, the lack of comparison between self-referential stimuli and other highly familiar stimuli sometimes precluded firm conclusions about the factors (i.e. self-reference or high familiarity) that caused attentional biases.

${ }^{3}$ We thank an anonymous reviewer for this suggestion.

${ }^{4}$ We thank an anonymous reviewer for pointing out this possibility.

\section{Acknowledgments}

This research was supported by a grant from the Belgian N.F.S.R. and a grant from NWO (Netherlands organization for Scientific Research), grant 402-01-630-PROG to JT. CD is a Postdoctoral Researcher at the Belgian N.F.S.R. We thank Gerry Altmann and three anonymous reviewers for their constructive comments on a previous version of this manuscript. 


\section{References}

Arnell, K.A., Shapiro, K.L., \& Sorensen, R.E. (1999). Reduced repetition blindness for one's own name: Evidence for conceptual representations. Visual Cognition, 6, 609-635.

Brédart, S., Delchambre, M., \& Laureys, S. (2006). One's own face is hard to ignore. The Quarterly Journal of Experimental Psychology, 59, 46-52.

Broadbent, D.E. (1958). Perception and Communication. London: Pergamon Press.

Bundesen, C., Kyllingsbaek, S., Houmann, K. J., \& Jensen, R. M. (1997). Is visual attention automatically attracted by one's own name? Perception \& Psychophysics, 59, 714720.

Deutsch, J., \& Deutsch, D. (1963). Attention: Some theoretical consideration. Psychological Review, 87, 272-300.

Fox, E., Russo, R., Bowles, R., \& Dutton, K. (2001). Do threatening stimuli draw or hold visual attention in sub-clinical anxiety? Journal of Experimental Psychology: General, 130, 681-700.

Gareze, L., \& Findlay, J.M. (2007). Absence of scene context effects in object detection and eye gaze capture. In R.P.G. van Gompel, M.H. Fischer, W.S. Murray, \& R.L. Hill. (Eds.), Eye movements: A window on mind and brain (pp. 617-637). Amsterdam, the Netherlands: Elsevier.

Gronau, N., Cohen, A., \& Ben-Shakhar, G. (2003). Dissociations of personally significant and task-relevant distractors inside and outside the focus of attention: A combined behavioral and psychophysiological study. Journal of Experimental Psychology: General, 132, 512-529.

Harris, C.R., Pashler, H.E., \& Coburn, N. (2004). Moray revisited: High-priority affective stimuli and visual search. The Quarterly Journal of Experimental Psychology, 57A, 131.

Kawahara, J., \& Yamada, Y. (2004). Does one's name attract visual attention? Visual Cognition, 11, 997-1017.

Kramer, A.F., \& Jacobson, A. (1991). Perceptual organization and focused attention : the role of objects and proximity in visual processing. Perception \& psychophysics, 50, 267284.

Laarni, J., Koljonen, M., Kuistio, A.M., Kyröläinen, S., Lempiäinen, J., \& Lepistö, T. (2000). Images of a familiar face do not capture attention under conditions of inattention. Perceptual and Motor Skills, 90, 1216-1218.

Lachter, J., Forster, K.I., \& Ruthruff, E. (2004). Forty-five years after Broadbent (1958): Still no identification without attention. Psychological Review, 111, 880-913.

Mack, A., \& Rock, I. (1998). Inattentional blindness. Cambridge, MA: MIT Press. 
Moray, N. (1959). Attention in dichotic listening: Affective cues and the influence of instructions. Quarterly Journal of Experimental Psychology, 11, 56-60.

Ro, T., Russell, C., Lavie, N. (2001). Changing faces: A detection advantage in the flicker paradigm. Psychological Science, 12, 94-99.

Shapiro, K.L, Caldwell, J., \& Sorensen, R.E. (1997). Personal names and the attentional blink: A visual "cocktail party" effect. Journal of Experimental Psychology: Human Perception and Performance, 23, 504-514.

Theeuwes, J., Kramer, A. F., Hahn, S., \& Irwin, D.E. (1998). Our eyes do not always go where we want them to go: capture of the eyes by new objects. Psychological Science, 9, 379-385.

Theeuwes, J., \& Van der Stigchel, S. (2006). Faces capture attention: Evidence from inhibition of return. Visual Cognition, 13, 657-665.

Tong, F., \& Nakayama, K. (1999). Robust representations for faces: Evidence from visual search. Journal of Experimental Psychology: Human Perception and Performance, $25,1016-1035$.

Underwood, G., Templeman, E., Lamming, L., \& Foulsham, T. (2008). Is attention necessary for object identification? Evidence from eye movements during the inspection of realworld scenes. Consciousness \& Cognition, 17, 159-170.

Weierich, M.R, Treat, T.A., \& Hollingworth, A. (2008). Theories and measurement of visual attentional processing in anxiety. Cognition \& Emotion, 22, 985-1018.

Wolford, G., \& Morrison, F. (1980). Processing of unattended visual information. Memory and Cognition, 8, 521-527.

Wood, N., \& Cowan, N. (1995). The cocktail party phenomenon revisited: How frequent are attention shifts to one's own name in an irrelevant auditory channel? Journal of Experimental Psychology: Learning, Memory, and Cognition, 21, 255-260. 